\title{
SPECTRAL ANALYSIS OF THE RELATIONSHIP BETWEEN ENERGY CONSUMPTION, EMPLOYMENT, AND BUSINESS CYCLES*
}

\author{
Umit EROL
}

Bilkent University, Ankara, Turkey

\author{
Eden S.H. YU
}

Louistana State University, Baton Rouge, LA 70803, USA

Received August 1988, final version received July 1989

\begin{abstract}
Through the use of cross-spectral techniques, we identify the dependence of U.S. industrial energy consumption on the industrial production index and total non-farm employment over business cycles. In particular, total energy consumption, mainly due to its industrial component, responds to changes in the production index. It is found that the industrial demand for energy is fairly sensitive to business cycles, while the household energy consumption and transportationsector energy consumption are not significantly affected by the expansion and contraction phases of the economy. In addition, there is a significant degree of correlation between the total non-farm employment and energy consumption over the business cycle frequencies. The evidence indicates a simultaneous response of employment and energy consumption to business cycle movements of the production index and suggests neutrality between energy and labor employment.
\end{abstract}

\section{Introduction}

Recently, the impacts of energy consumption on U.S. employment and gross national product (GNP) have been studied through the use of the traditional time-domain models. Applying Sims' (1972) technique, Kraft and Kraft (1978) found unidirectional causality running only from GNP to energy input for the period 1947-1974. By shortening the data sample by two years, Akarca and Long (1980) argued that this unidirectional causality appeared to be spurious. In a separate paper, Akarca and Long (1979) detected, by using Box-Jenkins time-series procedures, a unidirectional causality running from energy to employment. Their finding suggested a negative response of employment to a change in the energy input. Yu and Hwang (1984) found that although no causal relationship exists between

\footnotetext{
*The authors are indebted to Frederick Estabrook for drawing the diagrams. The usual caveat applies, however.
} 
GNP and energy, there was a slight unidirectional flow running from employment to energy. Energy conservation thus would lead to neither an increase nor a decrease in total employment. Recently, Erol and Yu (1987) using several time-series techniques discovered that there was no causal relationship between energy consumption and total employment.

The conflicting and inconsistent empirical results obtained for various sample periods by the above investigators may be attributable to the weakness associated with the time-domain models, especially the distributed lag models. The estimation of the time-domain models usually involves a series of trials, and the specification of the model is open to a certain amount of subjective judgment. ${ }^{1}$

An alternative approach to studying the properties of and the dynamic relationship between these energy and output (employment) series is the method of spectral analysis which analyses the series in the frequency domain. Over the past decade, spectral methods have been employed for examining several important economic issues [Scully $(1971,1974)$, Bohi and Scully (1975), Scully and Gallaway (1973)]. Undoubtedly, the spectral analytic approach has proved helpful in offering additional insight into various aspects of economic problems.

Both time-domain and frequency-domain procedures are non-structural approaches. They are thus useful in cases where a structural model is not transparent. Even though time-domain and frequency-domain are equivalent mathematically, their representational value is different. Each clarifies a different aspect of data. Frequency-domain sheds light on the degree of correlation at different frequencies, and especially important, on the type of relationship between the variables along the business cycles. This information may not be obtained from time-domain directly. Time-domain models require normally few parameters to be estimated given the parsimony principle in time-domain. Spectral domain, on the other hand, may require substantially more estimations of parameters. The choice is between parameterization of a model and the efficiency of estimation. Since we have already studied the time-domain properties of the relationship between energy and employment for the same observation period [Erol and $\mathrm{Yu}$ (1987)]. This study, a continuation of the research, aims at clarifying the frequency-specific nature of the relationship. We thus choose to apply the technique of spectral analysis to obtain additional insight into the issue.

The structural model of energy, employment and output is not transparent; the question of substitution and complementarity between factors of production and energy input has been debated but remains largely unresolved. $^{2}$ The primary purpose of this paper is to analyze the frequency-

\footnotetext{
${ }^{1}$ See Engle (1976) for an excellent discussion.

${ }^{2}$ See, for example, Berndt and Wood (1981), and Griffin (1981) for the exchange on the subject.
} 
domain characteristics of energy consumption and the industrial production index for the United States through the use of spectral and cross-spectral techniques. The industrial production index is classified as a coincident indicator and selected as an appropriate measure of business cycles. ${ }^{3}$ Insights into the dynamic relationships between energy and business cycles will be developed by testing several hypotheses. First, we will examine the question of the degree of the dependence or responsiveness of domestic total energy consumption to output changes over the business cycles. This responsiveness is best measured by the degree of correlation between the energy consumption and the industrial production index series. ${ }^{4}$ Second, we will study two additional issues concerning the responsiveness of various components of energy consumption to business cycle movements and the degree of correlation between total energy consumption and total non-farm employment. We expect that the industrial component of energy consumption to be responsive to the industrial production index.

The paper is organized as follows: Section 2 sketches the basic properties of spectral methods. This section can be skipped by those readers who are familiar with the techniques. Section 3 reports our investigation into the dynamic relationship between energy, output and employment by applying spectral analysis. Section 4 presents the concluding remarks.

\section{A synopsis of spectral techniques}

A fairly extensive formal literature has developed on the subject of spectral analysis. ${ }^{5}$ For an excellent heuristic discussion of the technique, the readers are referred to Scully (1971). Thus we sketch here only the most salient properties of spectral method. The spectral density function of a time series is related to the autocorrelation function of the same series by the following Fourier transformation:

$$
f(\theta)=\sum_{s=-\infty}^{\infty} R(s) \mathrm{e}^{-2 \pi i \theta}, \quad 0 \leqq \theta \leqq 1,
$$

where $R(s)$ is the autocovariance function. Eq. (1) shows the correspondence between the time-domain (e.g., ARMA) models and the frequency-domain models. Underlying both types of models is the assumption of stochastic

\footnotetext{
${ }^{3}$ For a related discussion, see Scully (1971, p. 367).

${ }^{4}$ Data sources: Monthly Energy Review, U.S. Department of Energy, various issues, and the U.S. Department of Commerce publications. The base year for the production index is 1967.

${ }^{5} \mathrm{~A}$ comprehensive treatment of the subject can be found in Jenkins and Watts (1968). Also see Granger and Hatanaka (1964), Fishman (1969) and Koopmans (1974)
} 
processes which are covariance stationary. While many economic time series violate the assumption of stationarity, it is possible to generate an approximately stationary series by taking the first differences of the variables. ${ }^{6}$

The spectrum is a decomposition of variance into the components contributed by each frequency. A peak in the spectral density function corresponds to a periodic component. A distinct peak in the spectrum identifies a reasonably regular cycle of the variable over the observation period. The cycle corresponds to a certain frequency band of the spectrum. ${ }^{7}$ A more interesting tool from the viewpoint of economic analysis is the crossspectrum which describes the relations between two stationary time series at different frequencies. The cross-spectrum is defined as

$$
f_{x y}(\theta)=\sum_{x=-\infty}^{\infty} \gamma_{x y}(\delta) \mathrm{e}^{-2 \pi i \theta s},
$$

where $\gamma_{x y}(\delta)$ is the cross-covariance function between the two series and $\mathrm{e}^{-2 \pi i \theta s}$ is the transformation operator. The properties of cross-spectrum can be quantitatively described in terms of statistics such as coherence squared, gain, and phase. The coherence squared is defined as

$$
\operatorname{COH}(\theta)=\left|f_{x y}(\theta)^{2}\right| / f_{x}(\theta) f_{y}(\theta),
$$

${ }^{6}$ The non-stationarity of economic time series poses additional specific problems in the spectral estimation. A trending mean results in a large peak at the first frequency band (at the zero frequency and very low frequencies). If the spectrum has a large peak at a certain frequency band, some of the power in this band may leak into the estimates of adjacent frequency bands, causing important bias in these estimates [Granger and Hatanaka (1964)]. This creates a specific problem in economic time series since the low-frequency band whose spectral estimates are affected describes the important business cycle activities. The coherence and phase estimates at low-frequency band are similarly affected. Various filtering methods are proposed in the literature to minimize this leakage. In this study, we have chosen to first-difference the natural logarithms of series in order to eliminate the trend of the series. This choice is suggested by the following reasons. The first is the fact that the time plots and autocorrelation functions showed that this operation transforms all of the series used in this study into an approximate stationary form. Also, the use of the same filter for all series is preferable from the viewpoint of preserving the validity of cross-spectral estimates. Our empirical trials with other filters such as a movingaverage type trend seasonal filter and modified Sim's filter showed that the general conclusions of this paper are not affected by the choice of filter; however, the coherence and phase values display some sensitivity to the choice of filter as expected. For a detailed discussion, see also Box and Jenkins (1976).

${ }^{7}$ The spectrum, in fact, is a plot of squared amplitude of each component against the frequency of that component. The squared amplitude measures the degree of contribution by variance to this frequency component. The frequency is related to the period of oscillation by the formula $f=2 \pi / T$ where $f$ is the radial frequency and $T$ is the period of oscillation [Jenkins and Watts (1968)]. 
where $\left|f_{x y}(\theta)^{2}\right|$ is the squared amplitude of cross-correlation between the two series, $f_{x}(\theta)$ is the spectral estimate of variable $x$, and $f_{y}(\theta)$ is the spectral estimate of $y$. Coherence is a measure of linear correlation between two variables at each frequency.

Two other statistics of interest are the gain and the phase statistics. The gain shows how much the spectrum of $x$ must be amplified to approximate that component of $y$ and is defined as

$$
G_{x y}(\theta)=\left|f_{x y}(\theta)\right| / f_{x}(\theta)
$$

The gain corresponds to the regression coefficient at a particular frequency; it is the regression coefficient at a particular frequency if any phase shift between the variables is eliminated. The phase measures the difference in time between the cycles of two variables and quantifies the lag at a certain frequency. The phase angle versus the frequency is plotted in the phase diagram. Phase angle is measured in terms of a fraction of a cycle that $y$ leads $x$ and is defined as

$$
P H(\theta)=\frac{1}{2_{\pi}} \arctan \left[-\operatorname{Im}\left(f_{x y}(\theta)\right) / \operatorname{Re}\left(f_{x y}(\theta)\right)\right]
$$

where $\operatorname{Im}\left(f_{x y}(\theta)\right)$ and $\operatorname{Re}\left(f_{x y}(\theta)\right)$ are, respectively, the imaginary and real parts of the spectrum. ${ }^{8}$

Both the spectrum and cross-spectrum are estimated from the sample data since the theoretical distribution is generally unknown. Statistical bias may result from improperly performed procedures. For example, the raw estimator (periodogram) is an inconsistent estimator. A number of tools can, however, be used to improve the reliability of the estimation procedure. One technique is the choice of a window with a selected maximal lag length in

\footnotetext{
${ }^{8}$ The phase diagram, theoretically, is a very valuable tool to analyze the lead-lag relationships at different frequencies. The practical applications of the phase diagram, however, poses certain problems. First, adding and subtracting one complete cycle from an angle will not change the tangent, and the phase can be known only up to adding or subtracting an integer [Engle (1976)]. This introduces some degree of ambiguity to the correct interpretation of the phase statistic. If the series have a mean phase whose values do not change with frequency, this may facilitate a more consistent analysis. Most economic time series however do not conform to this requirement and are characterized by phase estimates changing with frequency. Second, there is the problem of distortion of phase estimates by commonly used linear operations such as firstdifferencing [Koopmans (1974)].
} 
order to increase the resolvability of the spectrum. (Resolution is the ability of a spectrum to reveal the fine structure of the spectral and cross-spectral estimates.) In this study, the spectral statistics are obtained by using a triangular Bartlett type window with a maximal lag length of 40 months. ${ }^{9}$

Why is a window required for spectral estimation? The periodogram is an unbiased estimator but it is not consistent. Hence smoothing procedures called windows are required to ensure consistency. The width of the window is directly proportional with the number of points used in averaging. The spectral estimation in this study is done by the SAS-ETS package. The SASETS package basically allows the choice between rectangle or triangular windows. We have chosen a triangular (Bartlett) window in this study for the following reason: when there are distinct peaks in auto or cross spectrum (which is the case in this study), a rectangular window leads to bias in estimates and broadening of the peaks. A triangular window is better at describing the peaks since it attenuates the data away from the center of the data.

Regarding the width of the window used in this study, the triangular window chosen may be specified as $(1 / T)(1-|t| / T)$ if $|t| \leqq T$ and 0 if $|t|>T$ where $t$ is the number of time intervals and $T$ is the length of moving average lag or the width of the window.

We have estimated the spectra by using different windows (starting with a value of 10 and increasing the $T$ by 5 each time until $T=50$ ). The resulting choice of $T=40$ is an outcome of both the empirical trials and the following theoretical considerations. There is an unavoidable trade-off between efficiency and resolution in the window width choice. As we increase the window width, we gain in efficiency and the variance of the estimates is smaller. The cost is that the bias of smoothing becomes more serious and the degree of resolution diminishes. The efficiency problem is more important in our case since our sample is relatively small. In addition, there was no evidence of a substantial loss in resolution as we increased the window length from $T=5$ to $T=50$. In fact, the figures displayed in this study (all derived with a window width of $T=40$ ) show relatively well-defined peaks, and no evidence of a substantial broadening effect on peaks is visible. In other words, a gain in efficiency appears justifiable at the expense of a relatively less serious resolution loss in this case.

\footnotetext{
${ }^{9}$ Different types of windows have been proposed in the literature. In most cases, the choice of window is mainly dictated by computer package used. This was also the major factor in our choice of the window. It should be pointed out that empirical evidences do not favor one window over the others [Priestley (1981)]. The choice of maximal lag length is subject to some conflicting criteria. Increasing $M$ (the lag length) increases the resolvability of the spectrum but it also increases the variance of estimates [Grenander and Rosenblatt (1957)]. Jenkins and Watts (1968) suggests the use of a lag equal to $N / 3$ (where $N$ is the number of observations) to obtain the highest possible resolvability without leading to unacceptably large variances.
} 


\section{Dynamic relationships between energy and business cycles}

\subsection{Description of energy and its measurement}

Spectral and cross-spectral methods are applied to test the hypothesis of dependence of total and disaggregate energy consumption on output changes over the business cycle. Monthly data of various types of U.S. energy consumption and industrial production index covering the period January 1973 to December 1983 were used. Total or disaggregate energy demand in this study is compiled from Monthly Energy Review. Consumption of Energy is expressed in terms of quadrillion $\left(10^{15}\right)$ Btu. Total energy consumption is the sum of consumption of (a) coal, (b) natural gas (dry), (c) petroleum, (d) hydroelectric power (including industrial and utility production and net imports of electricity), (e) nuclear electric power, (f) net imports of coal coke, (g) others including geothermal power and electricity produced from wood, waste and wind energy. The data for the U.S. figure covers the consumption in 50 states and the District of Columbia.

The energy consumption of each type is expressed in terms of its appropriate unit. Coal consumption is measured in terms of thousand short tons. Consumption of coal in terms of thousand short tons is then converted into its appropriate heat content (measured in Btu) by using the conversion factors which are listed in the Appendix of Monthly Energy Review. Natural gas is measured in terms of billion cubic feet and is converted using similar conversion factors into its Btu equivalent. Petroleum consumption is the sum of several individual petroleum products consumed in each end-use sector, i.e., industry, household, electric utility and transportation. Individual petroleum products that are included in the total estimate are aviation gasoline, asphalt, distillate fuel, jet fuel, kerosene, LPG and lubricants. Consumption of individual petroleum products is measured in terms of thousands of barrels. The total estimate is then converted into its Btu equivalent.

Among the other energy types, hydro-electricity is measured in million kilowatt-hours, nuclear energy is measured in million net kilowatt-hours and geothermal energy is again in million kilowatt-hours. Each energy type is converted into its Btu equivalent heat content by using the appropriate conversion formula.

\subsection{Energy demand distribution}

The disaggregation of total energy consumption is given in terms of end-use sectors. Consumption by end-use sector follows the standard classification procedure used in government energy reports. The end-use sectors are primarily defined as residential and commercial (households), industrial and transportation. The disaggregated monthly figures of energy consumption are available only as consumption of these end-use sectors. The only further subdivision of data is a sub-division of each end-use sector consumption into 
coal, natural gas, petroleum, hydro-energy, etc. ${ }^{10,11} \mathrm{We}$ list the various data sources for energy consumption studies in the appendix. As is evident from the summary descriptions of the data sources, the available data are all presented in terms of household, industry and transportation end-use sectors. ${ }^{12}$ It may also be noted that the data on SIC level on a monthly basis and with sufficient number of observations to meet the requirements of spectral analysis are also unavailable.

For the present study, there are a total of 132 useful observations. The sample size appears somewhat limited, which may result in relatively wide confidence intervals for the coherence estimates; the estimates, however, are relatively consistent and unbiased with a sampe size of 100 observations or more [Jenkins and Watts (1968)].

\subsection{Cross-spectrum}

The first useful information is the cross-spectrum between total U.S. energy consumption and the production index for testing the dependence of energy to business cycle movements. Both series are expressed as the first difference of logarithms for transforming the series into stationary form by eliminating the observed trends in the available in the variables. (See footnote 6 for related discussion). We are aware of the issue of seasonality and of seasonal differencing. This issue was discussed in our earlier paper

\footnotetext{
${ }^{10}$ The energy consumption by residential and commercial units includes the energy consumption by private establishments (primarily for space and water heating, air-conditioning, and cooking), energy consumption by non-manufacturing business establishments, i.e., motels, restaurants, wholesale and retail companies, laundries, etc. and energy consumption by federal, state and local governments. The consumption of the industrial sector includes the energy consumed by manufacturing, construction, mining, agriculture and forestry. The consumption of the transportation sector includes energy consumed to move people and commodities in public and private sectors and pipeline transmission of natural gas.

${ }^{11}$ The only other possible sub-division of energy consumption in monthly figures involves sources of energy, e.g., total coal consumption, total gas consumption, etc. (and/or its further subdivision for each end-use sector). We choose to base this study on end-use sector classification for several reasons; first, the data required for the end use sector is readily available and the scope of the analysis is manageable. Second, and more importantly, the end-use approach is consistent with the main objectives of the present paper, which is to analyze the response of total energy consumption over the business cycles. Note that all the main indicators employed in the paper, i.e., production index, employment, energy consumption, etc., are quantity variables. In other words, this paper aims at establishing relationships between quantities.

${ }^{12}$ Conceptually, distribution of end-use sector energy demand can be made in terms of hosuehold, industry and transportation, or in terms of agriculture, commercial, service and government. The former classification is adopted in the present paper because the energy statistics reported by government follows such a classification scheme and, hence, the data for the end-use by household, industry and transportation are readily available. It may be, however, remarked that the distribution is partitioned among the distinctions used by standard allocation system. Thus, part of the government energy consumption is recorded as part of residential and commercial (household) consumption, while the energy consumed by public vehicles is recorded as part of the overall transportation sector energy consumption.
} 


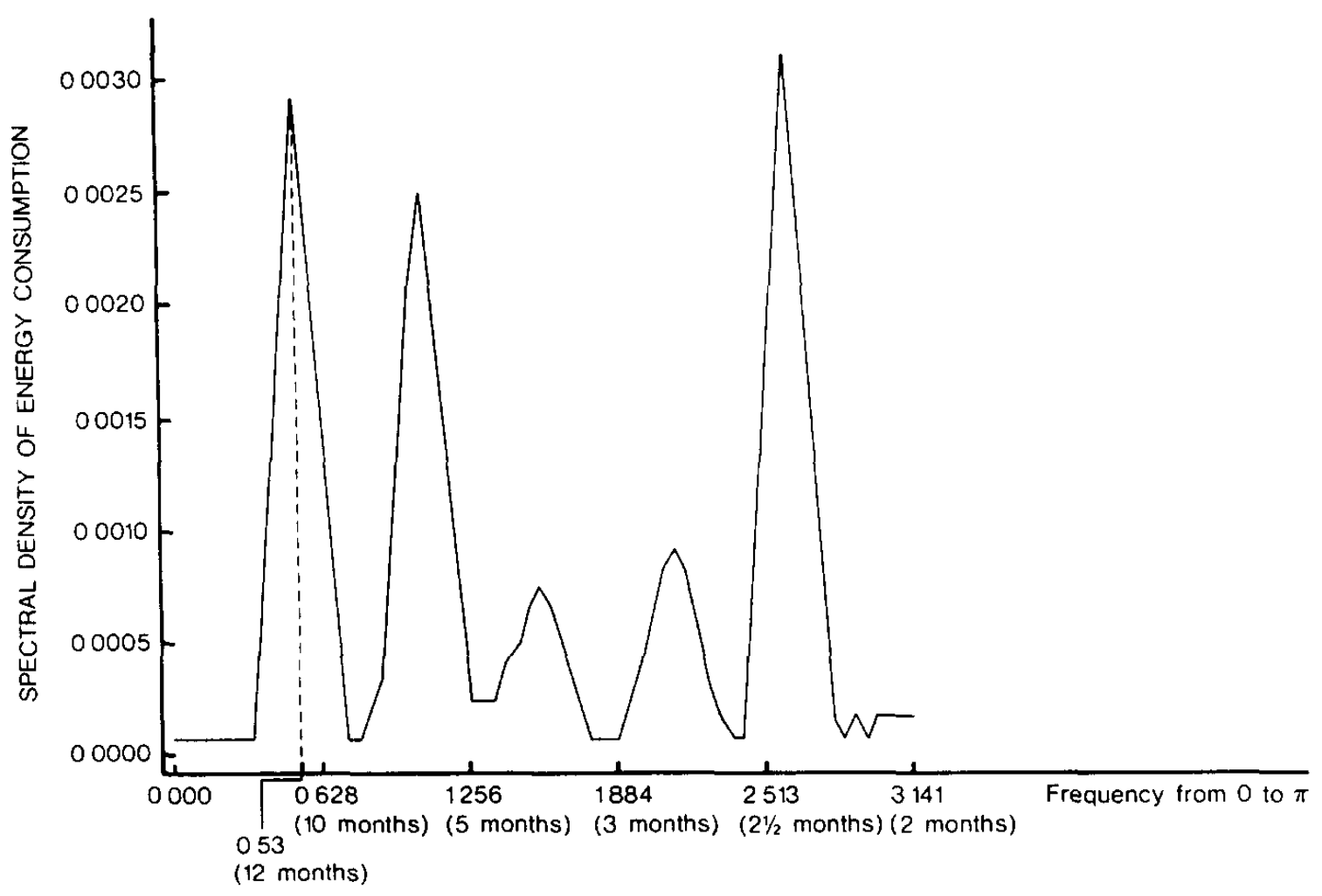

Fig. 1. The auto-spectrum of energy consumption for 1973-83.

[Erol and Yu (1987)]. However, seasonal differencing is not applied in this paper because the seasonality appears not strong enough to generate nonstationarity in the series. Neither the plot analysis nor the inspection of autocorrelation function clearly indicates non-stationarity in the series prior to seasonal differencing and after first-differrencing. Applying seasonal differencing is equivalent to applying a sharp filter to the frequency band centred at $f=1 / 2$. This poses a specific problem in spectral analysis. The sharp attenuation of spectrum at the seasonal frequency band usually leads to attenuation of nearby frequency bands due to leakage effect. In a small sample such as this, leakage effects causes distortion and attenuation in the adjacent business-cycle frequency range whose analysis is a paramount importance in this study. In fact, our suspicion on this possibility was confirmed when we attempted spectral estimation after seasonal differencing. The employment series, on the other hand, did not indicate any nonstationarity of this kind in our preliminary analysis. The same also applies to the industrial production index. The autospectrum of energy consumption is presented in fig. 1. The cross-spectrum between energy consumption and the production index is presented in fig. 2. The cross-spectral statistics are reported in table 1. We prefer to focus on the frequency bands that provide useful information about business cycles rather than to present all frequencies. The high-frequency bands (not shown in the tables), revealing a 


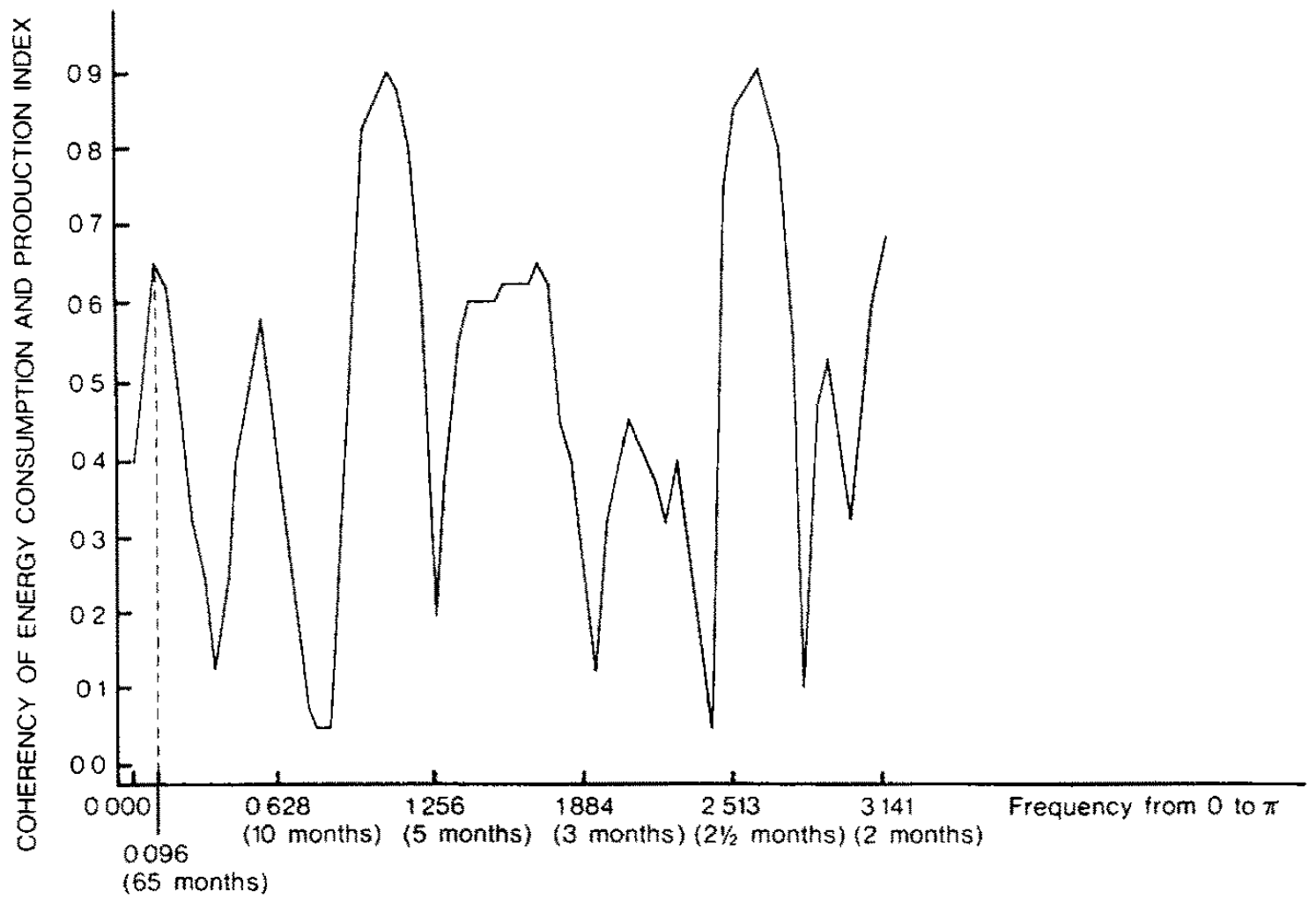

Fig 2. The cross-spectrum between the total energy consumption and production index.

Table 1

Cross-spectral statistics of the relation between the energy consumption and the production index..,

\begin{tabular}{cllll}
\hline Period & Frequency & Coherence & Phase & Gain \\
\hline 130 mon. & 0.048 & 0.50 & -0.19 & 0.771 \\
65 mon. & 0.096 & $0.65^{*}$ & -0.34 & 0.914 \\
43 mon. & 0.144 & $0.61^{*}$ & -0.42 & 0.956 \\
32 mon. & 0.193 & 0.51 & -0.55 & 0.927 \\
26 mon & 0.241 & 0.32 & -0.98 & 0.818 \\
21 mon. & 0.289 & 0.25 & -1.63 & 0.765 \\
18 mon. & 0.338 & 0.12 & -1.78 & 0.307 \\
16 mon. & 0.386 & 0.25 & -2.42 & 0.164 \\
14 mon. & 0.434 & 0.39 & -2.48 & 0.159 \\
13 mon. & 0.483 & 0.49 & -2.50 & 0.157 \\
12 mon. & 0.531 & $0.56^{*}$ & -2.53 & 0.165 \\
11 mon. & 0.579 & 0.47 & -2.52 & 0.171 \\
\hline
\end{tabular}

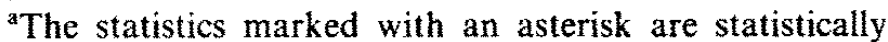
significant. The critical value for significance is approximately 0.53 at $95^{\circ} \%$ level with 10 degrees of freedom (Source: Amos and Koopmans tables, Amos and Koopmans, 1963).

bonly the implied tıme leads for statistically significant coherences are reported. No meaning can be ascribed to the time leads of statistically insignificant correlations. 
complicated pattern, are dominated by a series of peaks at the harmonics of the annual cycle and at Nyquist frequency. These frequencies are omitted because they do not convey any significant information from the viewpoint of economic analysis. Consequently, only the low-frequency bands corresponding to business cycles are reported. ${ }^{13}$

Granger and Morgenstern (1963) described the coherence as moderate if most coherences have the values between 0.3 and 0.6 , as high if most coherences are above 0.5 and some are above 0.8 , and as low otherwise. The coherences reported in table 1 clearly are in the moderate to high ranges. Stated differently, the cross-spectral analysis suggests a significant and strong correlation between the U.S. total energy consumption and the industrial production index. It is noteworthy that the highest correlation (squared coherence) between the series takes place at approximately 65 months, which roughly corresponds to a typical business cycle of five to six years. Since it is somewhat difficult to pinpoint the duration of periodic oscillations due to the changes in the duration of the business cycles, the interpretations of the periodic movements must rely on approximations rather than exact specifications. The view that there is a significant correlation between energy and output over the business cycle frequencies is also supported by the pattern of the gain statistics. The gain statistics attain their highest values between 32 and 65 months, which is the typical range of business cycles. The relatively high coherence $(0.56)$ at the 12 -month cycle and the adjacent frequencies indicate the presence of some seasonal correlation between the series.

\subsection{Stability issue}

Scully (1974) employed the gain and the phase statistics to determine if the inflation-unemployment trade-off was a static or dynamic relation. In static systems, the gain will be constant over all frequencies, and the phase will be zero or a pure delay lag or lead. In dynamic systems, the gain and the phase will be functions of frequency, and change significantly as the frequency changes. In the context of the present study, it is meaningful to consider the stability question of the relationship between energy consumption and the production index. Whether the relation is a static or dynamic one may be similarly inferred from the observation that static systems imply horizontal gain and phase functions and dynamic systems are characterized by sloped gain and phase diagrams. The gain and phase statistics presented in table 1 reveal that the relation between energy consumption and the production index can be chracterized as a dynamic relation rather than a static one, and the relation between the energy consumption and production index tends to

\footnotetext{
${ }^{13}$ The business cycles in economics are not represented by a single frequency point but by a frequency band. Granger and Hatanaka (1964) strongly advocates the study of whole frequency band ranging from 96 months to 12 months in order to capture the cyclical movements of the economy.
} 
shift in time. The sign of phase estimates is consistently negative over all the frequencies considered, indicating a lead of production index movements over energy consumption. The results clearly support the hypothesis that the energy consumption responds significantly to changes of production index over the business cycles.

\subsection{Disaggregate analysis}

As a secondary contribution, we test the degree of the dependence of various components of energy consumption on the production index over the business cycles. All the components are expressed in terms of the firstdifferences of logarithms. Table 2 presents the coherence statistics of household energy consumption, the energy consumption of the industrial sector, and the energy consumption of the transportation sector. The base index is again the production index, and the same set of observations is used. As in the previous case, seasonally unadjusted series are used. The coherence statistics indicate that the household energy consumption as expected does not significantly respond to business cycle movements; all the coherences over the business cycles are low and statistically insignificant (at the 95\% level). The relatively high coherence at the 12 -month cycle may only reflect a seasonal correlation with the production index. The energy consumption of the industrial sector, on the other hand, is somehow correlated with the production index. The highest coherence value in this frequency range is obtained at a cycle of 65 months as in the case of total energy consumption. The negative signs of the phase estimates indicate a lead of production index over the energy demand of the industrial sector, since the index of industrial production is not only a proxy for business cycles, but also a measure of the industrial sector's output. Thus, it is not surprising that this variable is found highly correlated with the energy use of the industrial sector. The apparent correlation between energy consumption and employment is mainly the result of the fact that both the employment and the industrial component of energy consumption respond to the cyclic changes of production index.

\subsection{Energy and total non-farm employment}

The significant correlation between the total energy consumption and the industrial production index reported earlier implies the possibility of a similar correlation between the energy consumption and the total non-farm employment over the business cycles. We are thus motivated to further test the degree of correlation between the energy consumption and the total nonfarm employment for the United States. Both series are prefiltered by taking the first differences of natural logarithms. The cross-spectral statistics are reported in table 3; the empirical results indicatee a significant degree of 
Table 2

Cross spectral statistics of the relation between the components of energy consumption and production index. $^{\mathrm{a}, \mathrm{b}}$

\begin{tabular}{lc}
\hline Period & Coherence \\
\hline$(A)$ Energy consumption of households \\
130 mon. & 0.21 \\
65 mon. & 0.33 \\
43 mon. & 0.22 \\
32 mon. & 0.13 \\
26 mon. & 0.05 \\
21 mon. & 0.01 \\
18 mon. & 0.03 \\
16 mon. & 0.15 \\
14 mon. & 0.32 \\
13 mon. & 0.47 \\
12 mon. & $0.56^{*}(-5$ mon.)
\end{tabular}

(B) Energy consumption of industrial sector

\begin{tabular}{cc}
130 mon. & 0.36 \\
65 mon. & $0.55^{*}(-6$ mon.) \\
43 mon. & 0.49 \\
32 mon. & 0.35 \\
26 mon. & 0.21 \\
21 mon. & 0.16 \\
18 mon. & 0.20 \\
16 mon. & 0.32 \\
14 mon. & 0.36 \\
13 mon. & 0.40 \\
12 mon. & 0.47 \\
(C) Energy consumption of transportation sector \\
130 mon. & 0.09 \\
65 mon. & 0.25 \\
43 mon. & 0.28 \\
32 mon. & 0.25 \\
26 mon. & 0.19 \\
21 mon. & 0.15 \\
18 mon. & 0.15 \\
16 mon. & 0.17 \\
14 mon. & 0.22 \\
13 mon. & 0.26 \\
12 mon. & 0.21 \\
\hline
\end{tabular}

${ }^{a}$ The coefficients marked with asterisk are statistically significant (See note a of table 1).

${ }^{b}$ The values in parentheses are time leads. Time leads are reported only for the statistically significant coefficients.

correlation between energy consumption and total non-farm employment over the business-cycle frequencies. The corresponding diagram (fig. 3) clearly shows a regular and well-defined peak at the business-cycle frequencies. The highest coherences of this frequency band are obtained between 65 and 32 
Table 3

Cross-spectral statistics of the relation between employment and energy consumption in the United States. ${ }^{\text {a.b }}$

\begin{tabular}{rllrl}
\hline Period & Frequency & Coherence & Phase & Gain \\
\hline 130 mon. & 0.04 & 0.49 & 0.06 & 0.224 \\
65 mon & 0.09 & $0.61^{*}$ & 0.01 & 0.240 \\
43 mon. & 014 & $0.68^{*}$ & 0.06 & 0.245 \\
32 mon. & 0.19 & $0.65^{*}$ & 0.01 & 0.236 \\
26 mon. & 0.24 & 0.40 & -0.26 & 0.181 \\
21 mon. & 0.28 & 0.11 & -0.89 & 0.097 \\
18 mon. & 0.33 & 0.01 & -0.88 & 0.018 \\
16 mon. & 0.38 & 0.03 & 1.92 & 0.010 \\
14 mon. & 0.43 & 0.09 & 1.83 & 0013 \\
13 mon. & 0.48 & 0.13 & 1.77 & 0.013 \\
12 mon. & 0.53 & 0.16 & 1.91 & 0.021 \\
11 mon. & 0.57 & 0.10 & 1.94 & 0.011 \\
\hline
\end{tabular}

${ }^{a}$ The coherences are statistically significant at approximately 0.53 (See note a of table 1 ).

b Only the time leads corresponding to significant coherences are.

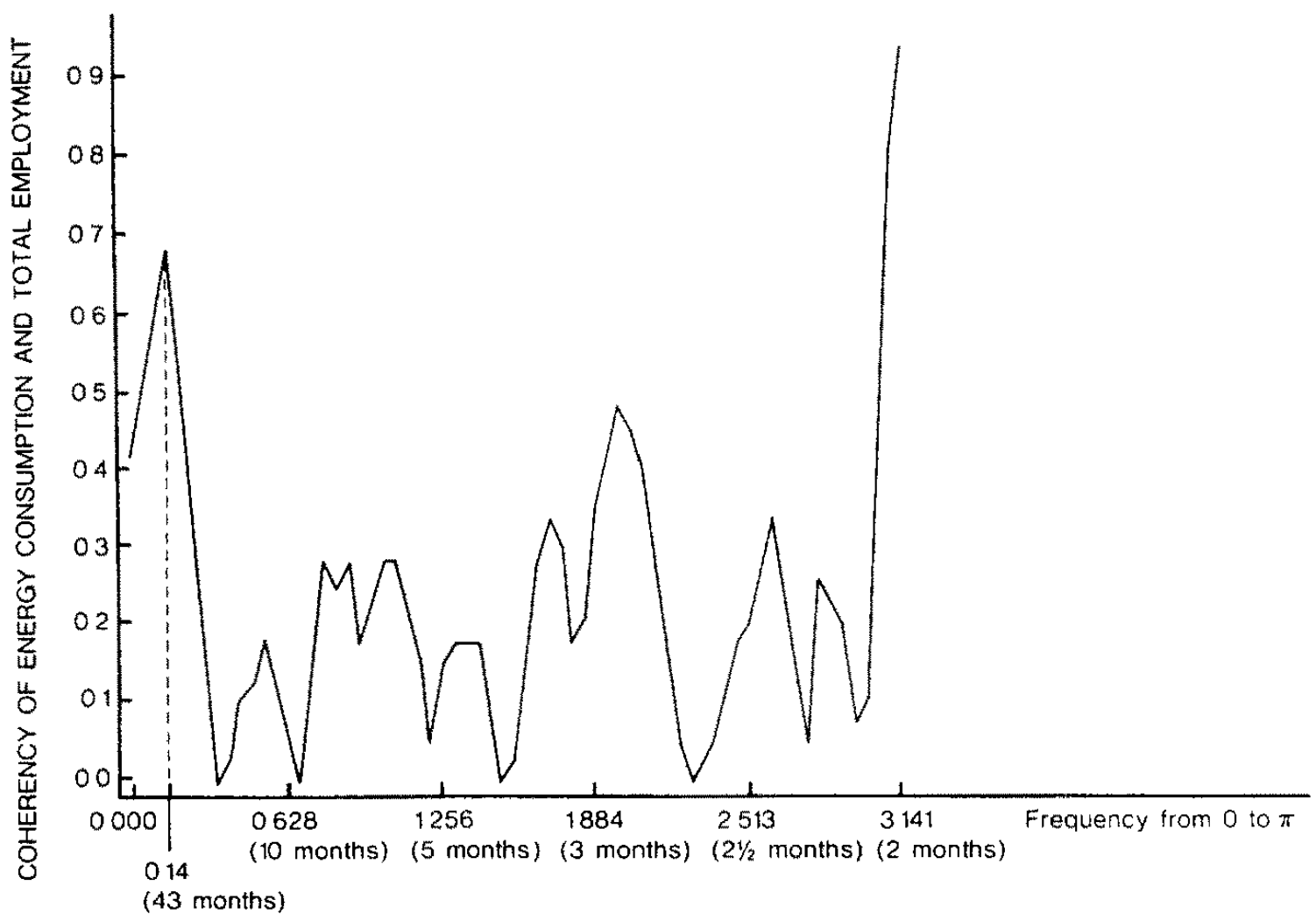

Fig. 3. The cross-spectrum between total energy consumption and total non-farm employment.

months (all over 0.6) which is the typical period for cyclic movements of the economy. The remaining peaks in the high-frequency band seem to indicate the harmonics of the fundamental cycles and a strong aliasing effect. There is 


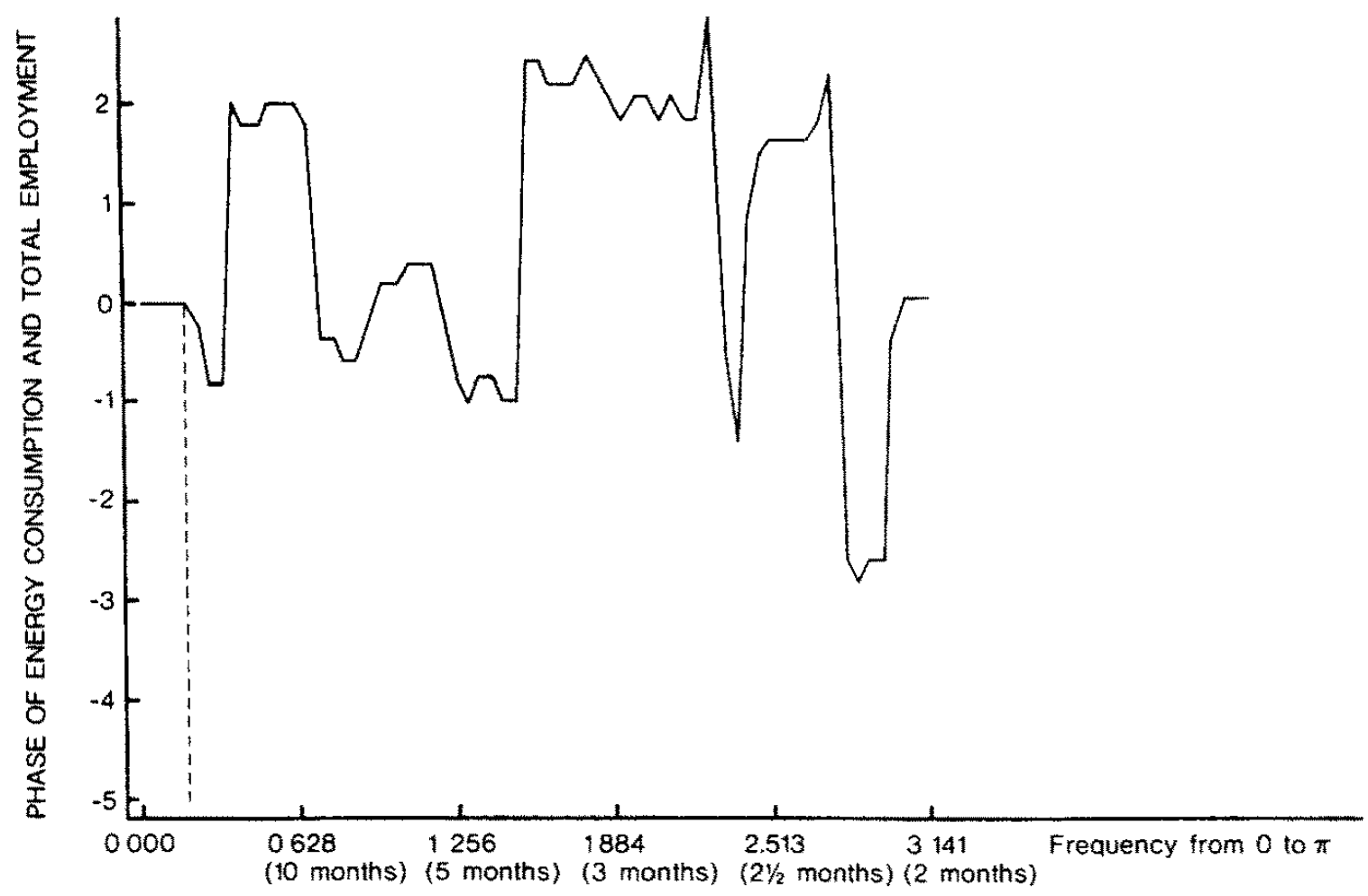

Fig. 4. The phase diagram of the relation between total energy consumption and total non-farm employment.

a further point that deserves attention. As elucidated by fig. 4 , which presents the phase diagram of the relation between the energy consumption and employment, it is noteworthy that, even though the overall phase diagram displays a changing pattern, the phase angles at the typical business-cycle frequencies are very close to zero. This strongly indicates that there is no time lead or lag between the series at this frequency band. This result is interesting from the viewpoint of suggesting a simultaneous response of employment and energy consumption to cyclic movements of the production index. The evidence seems to support the hypothesis that the observed linear correlation between energy consumption and employment is the result of a co-variation of both series with production index rather than showing a genuine causal relationship between employment and total energy consumption. This result is suggestive of the neutrality hypothesis. ${ }^{14}$

We have tested the causal relationship between the U.S. energy and employment for the same observation period [Erol and $\mathrm{Yu}$ (1987)]. We employed a number of alternative techniques of multiple time-series models to test the causality between U.S. energy and employment. All tests unanimously and clearly supported the neutrality hypothesis. This paper extends the previous research into frequency-domain. The spectral results

\footnotetext{
${ }^{14}$ The time-domain analysis by $Y u$ and Hwang (1984) strongly favored the neutrality hypothesis concerning the relationship between energy consumption and employment in a slightly shorter observation period.
} 
also favor the neutrality hypothesis. Fig. 3 shows that the only significant correlation between energy consumption and employment takes place at the low-frequency band corresponding to business-cycle frequencies. Fig. 4 indicates that the phase angle at this frequency range is conclusively a zero phase lag. This implies no lead or no lag between variables. It should be reminded that the Granger-causality criterion employs the concept of lead of $X$ over $Y$ to demonstrate causality. Then the seemingly contemporaneous correlation at the business cycle (with no lead) between the two variables suggest an indirect evidence for neutrality. This result and the stronger neutrality result obtained in time-domain strongly suggests that the observed dependence of energy consumption and employment on business cycles only indicates the co-movement of both variables with the production index without necessarily implying a causality between those variables in the Granger-sense.

\section{Conclusions}

Through the use of cross-spectral techniques, the dependence of the U.S. energy consumption on the industrial production index or the total non-farm employment over the business cycles has been identified. The primary empirical findings are as follows: (1) there exists a significant correlation between the total energy consumption (mainly due to the industrial component of energy consumption) and the production index over the typical business cycle frequencies; (2) the industrial demand for energy appears fairly sensitive to business cycles, while the household energy consumption and transportation-sector energy consumption are not significantly affected by the expansion and contraction phases of the economy; (3) there is a significant degree of correlation between the total non-farm employment and energy consumption over the business cycle frequencies. The evidence indicates a simultaneous response of employment and energy consumption to business cycle movements of the production index and is indirectly suggestive of neutrality between energy and labor employment.

\section{Appendix: Major data sources}

(1) Federal Reserve Monthly Statistical Releases: Is a monthly data source and presents indexes of industrial production and industrial electric power use.

(2) Coal Production - An EIA report: Is an annual data source reporting the coal production, prices, existing reserves and mining operations.

(3) Monthly Energy Review: Most data reported is compiled from other DOE (Department of Energy) publications. Contains monthly and final data. 
The classification is on the basis of standard classification of end-use sectors (residential and commercial, industrial and transportation).

(4) State Energy Data Report: Includes annual consumption estimates from 1960-83. The classification is on the basis of end-use sectors (resicential, commercial, industrial and transportation). Gives the energy consumption for each state on the basis of the above classification. Also the sources are elaborated (bituminous coal/lignite, anthracite, natural gas, etc.).

(5) State Energy Data Report Supplement: Gives the consumption estimates for 1960-83 period on an annual basis and includes energy consumption data by consuming sector on the basis of end-use classification scheme.

(6) Annual Energy Review: Presents the consumption of energy by end-use sector using the standard classification scheme of commercial and residential, industrial and transportation. The data is given on an annual basis and gives the energy consumed by households (by census region - data available for 1978-82); total individual sector energy consumption by source, petroleum products supplied to end-use sectors and also the consumption of natural gas, coal, electricity and wood energy by end-use sector.

(7) Energy Facts: Is an annual report presenting summary data on U.S. energy supply, demand and prices. Includes energy-consumption by end-use sector; refined petroleum products supplied to end-use sectors; natural gas delivered to customers and coal consumption by end-use sector. The disaggregation regarding the end-use sectors follows the standard classification scheme (residential and commercial, transportation and industrial).

(8) Residential Energy Consumption Survey: Is a survey repeated in every few years (the last one available is in 1983). It presents the national estimates of fuel consumption, household characteristics, type of fuel used by households. A similar survey report for non-residential building energy consumption can be found in Nonresidential Building Consumption Survey.

\section{References}

Akarca, A. and T.V. Long, 1979, Energy and employment: A time series analysis of the causal relationship, Resources and Energy 2, 151-162

Akarca, A.T. and T.V. Long, 1980, On the relationship between energy and GNP: A reexamination, Journal of Energy and Development 4, 326-331.

Amos, D.E. and L.H. Koopmans, 1963, Tables of the distribution of the coefficient of coherence for stationary bivariate Gaussian processes, Monograph SCR-483 (Sandia Corp.. Albuquerque, NM).

Berndt, E.R. and D.O. Wood, 1981, Engineering and econometric intepretations of energycapital complementarity: A reply and further results, American Economic Review 71, $1105-1110$.

Bohı, D. and G.W.A. Scully, 1975, Buyer's prices, seller's prices and price flexibility, American Economic Review 65, 517-525.

Box, G.E. and G. Jenkins, 1976, Time series analysis - Forecasting and control (Holden Day, San Francisco, CA). 
Engle, F.E., 1976, Interpreting spectral analysis in terms of time-domain models, Annals of Economic and Social Measurement 5, 89-101.

Erol, U. and E.S.H. Yu, 1987, Time series analysis of the causal relationships between U.S. energy and employment, Resources and Energy 9, 75-89.

Fishman, G., 1969, Spectral methods in econometrics (Harvard University Press, Cambridge, MA).

Granger, C.W.J. and M. Hatanaka, 1964, Spectral analysis of economic time series (Princeton University Press, Princeton, NJ).

Granger, C.W.J. and O. Morgenstern, 1963, Spectral analysis of New York stock market prices, Kyklos 16, 1-24.

Grenander. U. and M. Rosenblatt, 1957, Statistical analysis of stationary time series (Wiley, New York).

Griffin, J M., 1981, Engineering and econometric interpretation of energy-capital complementarity: Comment, American Economic Review 71, 1100-1104.

Jenkins, G.M and D.G. Watts, 1968, Spectral analysis and its applications (Holden Day, San Francisco, CA).

Koopmans, L.H., 1974, The spectral analysis of tıme series (Academic Press, New York).

Kraft, J and A. Kraft, 1978, On the relationship between energy and GNP, Journal of Energy and Development 3, 401-403.

Priestly, M.B., 1981, Spectral analysıs and time series (Academic Press, New York).

Scully, G.W., 1971, Business cycles and industrial strike activity, Journal of Business 44, 359-374.

Scully, G.W., 1974, Static vs. dynamic Philips curves, Review of Economics and Statistics 56, 387-393.

Scully, G.W. and L.E. Gallaway, 1973, A spectral analysis of the demographic structure of American unemployment, Journal of Business 42, 87-102

Sims, C.A., 1972, Money, income and causality, American Economic Review 62, 540-552.

Sims, C.A., 1977, Comment on Pierce, Journal of American Statistical Association 72, 23-24.

Yu, E. and B.K. Hwang, 1984, On the relationship between energy and GNP: Further results, Energy Economics 6, 186-190. 